\title{
Let MultiBand-OFDM Modified By Wavelet
}

\author{
J. Avila* and K. Thenmozhi \\ School of Electrical \& Electronics Engineering, SASTRA University, Thanjavur, \\ Tamil Nadu-613401, India; avila@ece.sastra.edu
}

\begin{abstract}
This paper throws light on elevating the performance of MB-OFDM by diverse techniques. The initiative was that the wavelet transform altered the place of FFT and IFFT. The use of the wavelet transform can be ascribed to the fact that it counteracts various types of interference. Implementation of various modulation techniques refines the performance. The order of the wavelet transforms used should remain conducive to establish it as an efficient system for high data rates. As an outcome of noise the output of the channel is erroneous. Hence error control codes are utilized which aims at the removal of error from the transmitted bits. Further to achieve diversity space time block codes are concatenated with FEC codes.
\end{abstract}

Keywords: Fast Fourier Transform (FFT), Multiband OFDM, Neural Network, Space Time Block Code (STBC), Ultra Wide Band, Wavelet Transform

\section{Introduction}

The ultra wide band spectrum extending from $3.1 \mathrm{GHz}$ to $10.6 \mathrm{GHz}$ faces difficulty in its handling owing to its wide spectral width ${ }^{1}$.To circumvent the challenges multiband OFDM came into its application by virtue of its high data rates, less power consumption and need of less silicon space. The frequency plan of UWB shows the division of the entire spectrum into 5 master groups ${ }^{2}$, each holding 3 sub-bands with an exception of the last group with 2 sub-bands making a total of 14 . Among the 128 available sub-carriers with sub-carrier spacing of $4.125 \mathrm{MHz} 100$ is used for data transmission, 12 plays the role of the pilot carriers ${ }^{3}, 10$ as guard tones and 6 as null tones.

The block diagram enumerating the MB-OFDM is shown in Figure 1. The input data sequence is taken randomly with the help of a scrambler followed by encoding the channel using an encoder ${ }^{4}$. The multipath fading of the communication channel is corrected using suitable error control codes. During the presence of heavy noise, concatenation of such codes is implemented to increase the coding gain and efficiency of the system ${ }^{5}$. The encoded bits then find its way through a 3-stage interleaver which

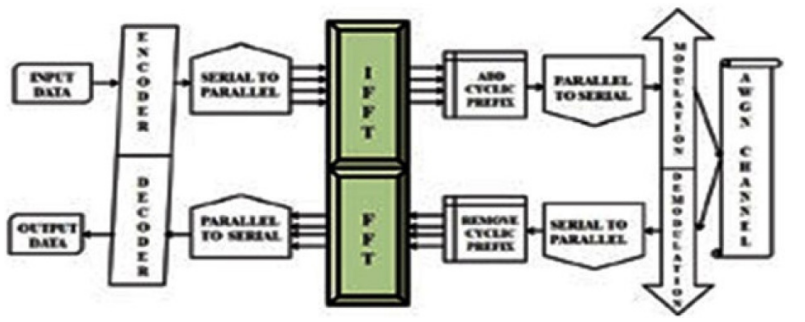

Figure 1. Block diagram of Multiband OFDM.

provides stability against burst error. The bits are then mapped into constellation samples ${ }^{6}$. The mapped output is sent through an IFFT block to convert into OFDM symbols by an OFDM modulator. The receiver implements the reverse operations such as demodulation and decoding to retrieve the original sequence ${ }^{7}$.

To ensure reliable communication error control codes are added along with the message Reed Solomon codes, being non-binary depend on Galois field and its number of bits in a system is determined by its characteristics. Sometimes parity bits are added to the data blocks. The relation $2 \mathrm{x}=\mathrm{Y}-\mathrm{l}^{8}$ highlights its error correcting capability wherein $\mathrm{Y}$ represents the length of the code and 1 repre-

${ }^{*}$ Author for correspondence 
sents the message. The limit to the maximum length of the code is valued by $2^{\mathrm{c}}-1$ where $\mathrm{c}$ is the number of bits.

Similarly, the turbo codes are implemented by serial or parallel connections with block codes or convolution codes and performance depends on decoding algorithm; interleave size and constraint length' ${ }^{9}$. Soft decision decoding is performed at the receiver side to yield better results.

The implementation of binary phase shift keying was taken owing to its simplicity and robustness ${ }^{10}$. This scheme sounds less sensitive to noise as the distance between the points is found to be higher leading to inefficient bandwidth utilization. The transmitted signal is given by

$$
\begin{aligned}
& D(t)=\sqrt{2 R_{b} / D_{b}} \cos (2 \pi b t) ; 0 \leq t \leq D_{b} \text { for binary one } \\
& D(t)=-\sqrt{2 R_{b} / D_{b}} \cos (2 \pi b t) ; 0 \leq t \leq T_{b} \text { for binary one } \\
& K(t)=p(x) \sqrt{2 R_{b} / D_{b}} \cos (2 \pi b t)
\end{aligned}
$$

where,

$p(x)= \pm 1$

$D_{b}$ is the bit period

$R_{b}$ is the energy

$b$ is the carrier frequency

The probability of error is given by

$$
\mathrm{P}=1 / 2 \operatorname{erfc} \sqrt{D_{b} / M_{0}}
$$

Quadrature Amplitude Modulation (QAM) makes use of both the techniques namely Phase Shift Keying (PSK) and Amplitude Shift Keying (ASK) ${ }^{11}$. The type of QAM to be used is based upon the application

$$
\begin{aligned}
Z(t)= & d_{o}(t) \sqrt{E} \cos \left(2 \pi f_{0} t\right) \\
& +d_{e}(t) \sqrt{E} \sin \left(2 \pi f_{0} t\right)
\end{aligned}
$$

where,

$\mathrm{E}=$ amplitude of the signal

$\mathrm{d}_{\mathrm{o}}(\mathrm{t})=$ odd number sequence

$\mathrm{d}_{\mathrm{e}}(\mathrm{t})=$ even number sequence

Quadrature phase shift keying has a phase change of 90 degree between the binary values. It supports high data rate when compared with BPSK and less error prone when compared with QAM.

In the proposed work Coiflet wavelet transform ${ }^{12,13}$ is utilized in MB-OFDM system. This wavelet family has orthogonality property similar to Fast Fourier Transform. Hence it becomes a fruitful alternate to FFT in the multiband OFDM system. In addition its characteristics are compared with the presence of three modulation schemes namely Binary Phase Shift Keying
(BPSK), Quadrature Phase Shift Keying and Quadrature Amplitude Modulation (QAM).

Further to combat heavy noise and to achieve diversity the error control codes namely convolutional code, turbo code and Reed Solomon code are concatenated with Alamouti code. Alamouti code is preferred because it is simpler and less complex one in the STBC family. Finally the encoder and decoder of the multiband OFDM system is trained using neural network. Backpropagation algorithm is utilized in the study to achieve accurate results.

\section{Materials and Methods}

The IFFT and FFT blocks in the transmitter and receiver are changed by IDWT and DWT block respectively. Wavelet transform has the capability to work under both the time and frequency domains. This feature makes it more popular and has a wide range of applications in many fields like image processing, signal processing etc. It is basically a filtering operation. The wavelet tree is complied by dividing the incoming signal into low and high frequency components. High frequency components offers accurate results and low frequency components offer approximate output14. The block diagram of modified Multiband OFDM is as shown in the Figure 2.

Haar wavelet is of maximized implantation subjected to its simplicity and fast nature. Though it is two elements wide it is not applicable for audio applications ${ }^{15}$. Wavelet coefficients are obtained by reversing the order of scaling coefficients and changing the sign of the second term ${ }^{16}$. The scaling and wavelet functions could be obtained from the inner product of data and coefficients. Symlet wavelet transform are modified version of Daubechie. They are more symmetric and have even index number. In addition they have minimal phase ${ }^{17}$. Coiflet wavelet is more symmetric than the above wavelets.

\subsection{Neural Networks}

A neural network is an analogy to the human brain. The neural networks are characterized by its interconnections

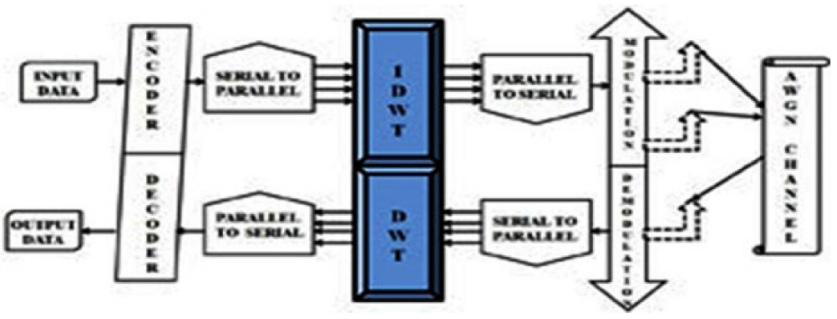

Figure 2. Block diagram of modified Multiband OFDM. 
called processing units whose functionality is similar to that of the neurons in the brain ${ }^{18}$. The neural network aims at the prediction in the happening of uncertain events. The proposed structure undergoes supervised learning and is trained to a given set of input patterns to a predefined target. Among the various training algorithms that is present the back propagation algorithm is chosen as it minimizes the error by back propagating it to the input. This feature is highly useful in networks devoid of feedbacks ${ }^{19}$.

One of the algorithms prevailing in the field of neural networks is the back-propagation algorithm, popularly called as BPN which finds application including pattern recognition and classification. The BPN algorithm allows the feed forward neural network to have multiple layers and back propagate (i.e. Retrace) the error from the output to the input through the hidden layers thus facilitating correction mechanisms. The use of non-linear activation function gives better accuracy in convergence and it is faster than Jacobi's method. This algorithm makes the neural network converge for the most precise output even for a noisy input ${ }^{20}$. Figure 3 shows the block diagram of ANN trainer. Here two layers are used. BPN algorithm is used for training and for training the parameters are set as per Table 1.

Alamouti scheme being the simplest in the Space Time Block Codes (STBC) family is implemented to achieve diversity. It is a two transmitter and one receiver scheme.Alamouti code concatenated with error control coder results in enhanced version of the system

\section{Results and Discussion}

In this research contribution all the graphs are plotted using MATLAB. Results are concluded from the Bit Error Rate (BER) versus $\mathrm{Eb} / \mathrm{N}_{0}$ curve plotted for the multiband OFDM system.

Figure 4 shows the output of Reed Solomon code concatenated with Alamouti code with FFT being replaced

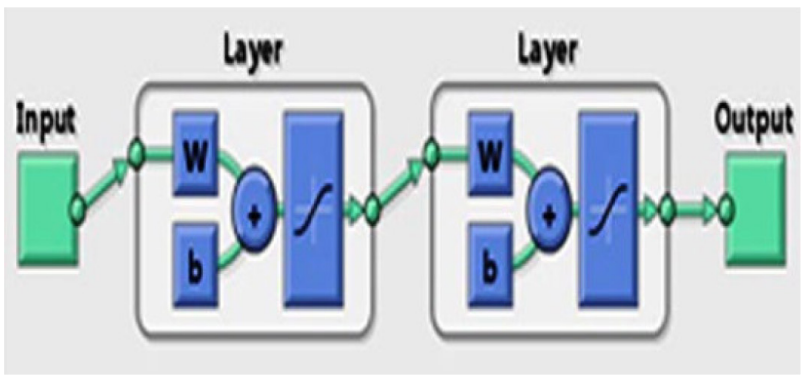

Figure 3. Block diagram for ANN Encoder and Decoder. with wavelet families Haar and Coiflet. BPSK modulation scheme is preferred because it is less error prone when compared with other schemes. In both the cases for a given BER of $10^{-4}$ the concatenated code overrules the individual code because of the increased coding gain and improved diversity.

Figure 5 shows the output of the convolutional code concatenated with Alamouti code for QPSK modulation and with coiflet family. The combination of convolutional code with QPSK is more suitable for wireless communication. For a given BER of $10^{-5}$ there is atleast $1 \mathrm{~dB}$ difference between concatenated code and the individual one.

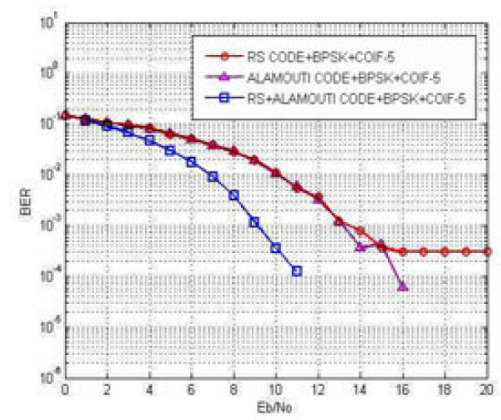

Figure 4. RS code concatenated with Alamouti code.

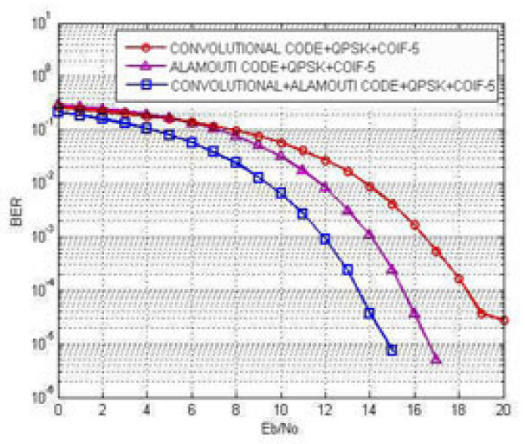

Figure 5. Convolutional code concatenated with Alamouti code.

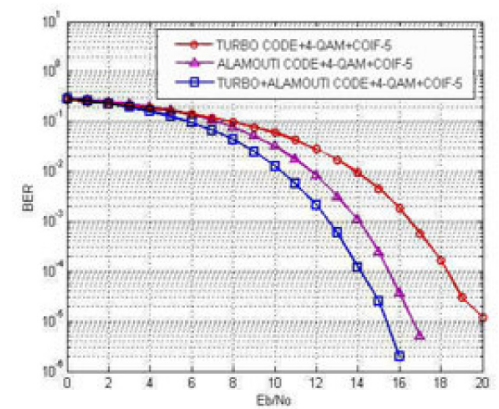

Figure 6. Turbo code concatenated with Alamouti code. 
Figure 6 shows the output of the turbo concatenated with Alamouti code with FFT being replaced with wavelet family named Coiflet. In this for a given BER of $10^{-5}$ the concatenated code offers better results when compared with individual code. When compared to other error control codes turbo codes offer good BER performance. Hence QAM scheme is preferred here to support high data rates. This combination in line deepens the quality of the multiband OFDM system.

Figure $7 \mathrm{a}$ and $7 \mathrm{~b}$ shows the encoder and decoder output of multiband OFDM trained using a Back Propagation algorithm respectively. The training sequence is performed based on the parameters given in table 1. The BER is given as target and the corresponding output is given as input to the algorithm and it is trained. The intersection circle in the figure shows that the target is reached.

\section{Conclusion}

In this work the enactment assessment of DWT based MB-OFDM is carried out for Coiflet wavelet families.
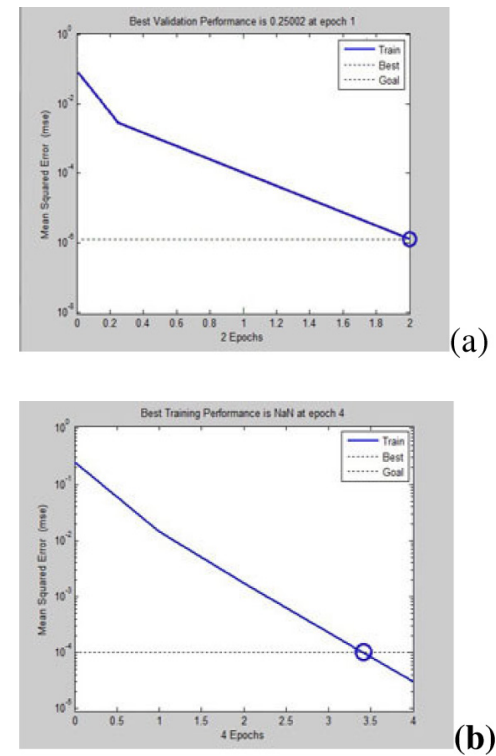

Figure 7. (a) ANN trained encoder, (b) decoder part of multiband OFDM.

Table 1. Neural network training parameters

\begin{tabular}{lc}
\hline Parameters & Back propagation algorithm \\
\hline Number of layers & 3 \\
Transfer function & Transig \& logsig \\
Training Epoch & 420 \\
Learning rate & 0.05 \\
Training goal & $10^{-4}$ for encoder, $10^{-6}$ for decoder \\
\hline
\end{tabular}

Convolutional code, Turbo code and Reed Solomon codes concatenated with Alamouti code are used to combat the channel noise and finally the analysis is also carried out between the modulation schemes namely BPSK, QPSK and QAM where the former is less prone to error, QPSK is moderate one and later supports high data rates. Proper choice of constellation mapper is necessary based on the application. All these techniques (wavelet, FEC, constellation mapper) make MB-OFDM a perfect solution for high data rates. The encoder and decoder part of the multiband OFDM system is trained using neural network.

\section{References}

1. Balakrishnan J, Batra A, Dabak A. A multiband OFDM system for UWB communication. IEEE Conference on Ultra Wide Band Systems and Technology; 2003. p. 354-58.

2. Praveenkumar P., Amirtharajan R., Thenmozhi K, Rayappan JBB. Regulated OFDM-role of ECC and ANN-A review. J Appl Sci. 2012; 12 4:301-14.

3. Avila J, Vinoth E, Thenmozhi K. Anti Interference for anti corroupt for $3 \mathrm{G}$ spectrum-A multi carrier approach. IEEE International Conference on Computer Communication and Informatics; 2012. p. 478-81.

4. Thenmozhi K, Padmapriya V, Amirtharajan R, Prithiviraj V, Varadarajan R, Rayappan JBB. OFDM + CDMA + Stego = A Secure Communication: A review. Res J Inform Tech. 2012; 4 4:31-46.

5. Avila J, Vinoth E, Praveen E, Thenmozhi K. Performance augument of Multiband OFDM with ANN assisted concatenated codes. IEEE International Conference on Computing, Electronics and Electrical Technologies; 2012. p. 76-80.

6. Batra A, Lingam S, Balakrishnan J. Multiband OFDM: A cognitive radio for UWB. Proceedings of International symposium on circuits and systems; 2006. p. 4094-97.

7. Avila J, Praveen E, Varadharajan B. ANN assistedAugumentation of AIC for MIMO Multiband OFDM System. IEEE International Conference on Advances in Engineering, Science and Management; 2012. p. 228-32.

8. Viraktamath SV, Attimarad GV. Impact of constraint length on performance of convolutional CODEC in AWGN channel for image application. Int J Eng Sci Tech. 2010; 2(9):4696-700.

9. Hoosier R, Jamali SH, Bahai ARS. Turbo coding performance in OFDM packet transmission. IEEE Vehicular Technology Conference Proceedings. 2000; 2(2): 805-10.

10. Kumar S, Sharma S. Error probability of different modulation schemes for OFDM based WLAN standard IEEE 802.11a. Int J Eng Educ. 2010; 4(4):262-67. 
11. Sklar B, Ray PK. Digital communication-Fundamentals and applications. 2nd edn. Pearson Education.

12. Gupta D, Vats VB, Garg KK. Performance analysis of DFT -OFDM, DCT-OFDM and DWT-OFDM in AWGN channel. International Conference on Wireless and Mobile Communications; 2008. p. 214-16.

13. Das B, Tiwari S, Das S. Performance study of discrete wavelet packet based MB-OFDM system for short range indoor wireless environment. IEEE International Conference on Devices and Communication; 2011. p. 1-5.

14. Alwadi S, Ismail MT, Karimal SAA. Discovering Structure breaks in Amman stocks market. Journal of Applied Science. 2011; 117:1237-78.

15. Mahmoud MI, Deyab MIMS, Elfouly FH. Comparison between Haar and Daubechies Wavelet Transformations on FPGA Technology. World Academy of Science, Engineering and Technology. 2007; 203:68-72.
16. Idi BY, Kamarudin MDN. Interpretation of ground penetrating Radar Image using discrete wavelet transform. Asian Journal of Applied Sciences. 2012; 53:174-82.

17. Chavan MS, Mastorakis N, Chavan MN, Galkwad MS. Implementation ofSYMLET Wavelets to removal of Gaussian additive noise from speech signal. Tenth International conference on Recent Researches in Communications, Automation, Signal Processing; 2011. p. 37-41.

18. Kumar V. Neural Network \& Fuzzy Logic Control, Lakshmi Publications. Second edn; 2008.

19. Tumuluru VK, Wang P, Niyato D. A neural network based spectrum prediction scheme for cognitive radio. IEEE International Conference on Communications. 2010; 23-7.

20. Freeman JA, Skapura DM. Neural networks algorithms, applications, andprogrammingtechniques.PearsonEducation; 2002. 